\title{
The Effects of Galactic Winds on the Chemical Evolution of Baryons
}

\author{
María E. De Rossi ${ }^{1,2}$ \& Patricia B. Tissera ${ }^{1,2}$ \\ ${ }^{1}$ Instituto de Astronomía y Física del Espacio, CONICET-UBA, \\ CC67 Suc28, Ciudad Autónoma de Buenos Aires (1428), Argentina \\ email: derossi@iafe.uba.ar \\ ${ }^{2}$ Consejo Nacional de Investigaciones Científicas y Técnicas, \\ Rivadavia 1917, Ciudad Autónoma de Buenos Aires (1428), Argentina
}

\begin{abstract}
During the last decade, the development of more sophisticated surveys allows the exploration of the chemical enrichment of galaxies and their environment at different cosmic times. In this context, it has been suggested that galactic winds play a fundamental role in the regulation of the star formation and the transportation of metals outside the galaxies. In this work, we study the influence of Supernovae winds in the chemical evolution of galaxies and the intergalactic medium by employing cosmological numerical simulations within a hierarchical Universe. We focus on the origin and evolution of the mass-metallicity relation (MZR).
\end{abstract}

Keywords. Galaxies: formation, Galaxies: evolution, Cosmology: dark matter

\section{Introduction}

In the Local Universe, there is a clear correlation between the stellar mass and chemical enrichment of galaxies in the sense that brighter and more massive systems exhibit higher metallicities (e.g. Tremonti et al. 2004). Moreover, the MZR seems to evolve with time in such a way that, at a given stellar mass, galactic systems were less enriched in the past (e.g. Maiolino et al. 2008). Tissera et al. (2005) and De Rossi et al. (2007, DR07) studied numerical simulations within a $\Lambda$-CDM cosmology showing the fundamental role of the hierarchical building up of the structure on the origin of the MZR. Here, we extend that work by studying the influence of galactic winds on the chemical enrichment of baryons and the evolution of the MZR.

\section{Simulations and galaxy sample}

We performed numerical simulations consistent with the concordance $\Lambda$-CDM universe with $\Omega_{\mathrm{m}}=0.3, \Omega_{\Lambda}=0.7, \Omega_{\mathrm{b}}=0.04$ and $\mathrm{H}_{0}=100 h^{-1} \mathrm{~km} \mathrm{~s}^{-1} \mathrm{Mpc}^{-1}$ with $h=0.7$. A version of GADGET-3 including treatments for metal-dependent radiative cooling, stochastic star formation, chemical enrichment and Supernovae (SN) feedback (Scannapieco et al. 2006) was used. We adopted a Salpeter Initial Mass Function with lower and upper mass cut-off of $0.1 M_{\odot}$ and $40 M_{\odot}$, respectively. The simulated volume corresponds to a cubic box of a comoving $10 \mathrm{Mpc}^{-1}$ side length. The simulation has a mass resolution of $5.93 \times 10^{6} M_{\odot} h^{-1}$ and $9.12 \times 10^{5} M_{\odot} h^{-1}$ for the dark and gas phase, respectively.

Galactic systems were identified by combining a friends-of-friends technique with the algorithm SUBFIND (Springel et al. 2001). The mean properties of galaxies were estimated at the optical radius $\left(R_{\text {opt }}\right)$, defined as the one which encloses 83 per cent of the baryonic mass of the systems. 


\section{Results and Discussion}

Our simulations predict a clear correlation between stellar mass and gas-phase chemical abundances from $z \approx 3.5$. Moreover, the simulated MZR exhibits similar patterns to the observed one. There is a decrease in the zero point value by approximately a dex with respect to the model without galactic winds described by DR07, showing the efficiency of SN winds in ejecting metals outside the central regions of galaxies. This decrease in the metal content of simulated galaxies allows us to better describe the observed correlation at high redshifts. However, as there is no observational consensus about the exact value of the zero point of the MZR, it is more robust to study the level of evolution. In this sense, at a given stellar mass, the model which includes SN feedback predicts a weaker evolution than what is suggested from observations. In fact, although the feedback model is able to better describe the dynamical and chemical enrichment of galaxies, the galactic winds generated in this simulation seem to be very efficient, producing an important decrease in the chemical content of galactic systems.

Contrary to observations, DR07 found larger effective yields for smaller galaxies at $z \sim 0$, which suggests the need for strong SN outflows in the model. In this work, we verify that the treatment of galactic winds addresses the problem of the slope of the yieldmass relation in the Local Universe (De Rossi et al. 2010, in preparation). However, the predicted yields are lower than the observed ones, consistently with the trend obtained for chemical abundances.

We also studied the gas fraction-stellar mass relation as a function of redshift. Although there is an important dispersion, we obtained no significant evolution of the mean gas fractions with time in contradiction with observations (e.g. Erb et al. 2006). These results indicate that SN feedback is very efficient at heating up the gas, inhibiting the star formation process. These conclusions are consistent with the weaker evolution obtained for the MZR. Nevertheless, it is worth noting that simulated gas fractions at $z=0$ are consistent with observations in the Local Universe (e.g. Zhang et al. 2009).

Our results indicate that SN feedback efficiency needs to be revised by varying the parameters of the SN model in order to reproduce the observed level of evolution of the MZR. Nevertheless, we are in the right way as this model is able to describe the observed features of the MZR at high redshifts and reproduce the dependence of yield on mass in the Local Universe. Moreover, the SN feedback model is able to describe the formation of disk galaxies and predicts a correlation between stellar masses and circular velocities in agreement with the observed Tully-Fisher Relation (De Rossi et al. 2009, in preparation).

\section{Acknowledgments}

We are grateful to CONICET, PICT 32342 (2005) and PICT 245 (2006). The authors would like to thank the IAU for its financial support to attend this meeting.

\section{References}

de Rossi, M. E., Tissera, P. B., \& Scannapieco, C. 2007, MNRAS, 374, 323

Erb, D. K., et al. 2006, ApJ, 646, 107

Maiolino, R., et al. 2008, A\&A, 488, 463

Scannapieco, C., Tissera, P. B., White, S. D. M., \& Springel, V. 2006, MNRAS, 371, 1125

Springel, V., White, S. D. M., Tormen, G., \& Kauffmann, G. 2001, MNRAS, 328, 726

Tissera, P. B., De Rossi, M. E., \& Scannapieco, C. 2005, MNRAS, 364, L38

Tremonti, C., et al. 2004, ApJ, 613, 898

Zhang, W., et al. 2009, MNRAS, 397, 1243 